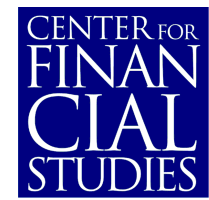

No. $2003 / 02$

\title{
On the Relation between Robust and Bayesian Decision Making
}

Klaus Adam

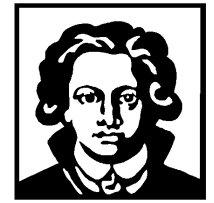

Center for Financial Studies 


\title{
On the Relation between Robust and Bayesian Decision Making
}

\author{
Klaus Adam*
}

December 2002

\begin{abstract}
:
This paper compares Bayesian decision theory with robust decision theory where the decision maker optimizes with respect to the worst state realization. For a class of robust decision problems there exists a sequence of Bayesian decision problems whose solution converges towards the robust solution. It is shown that the limiting Bayesian problem displays infinite risk aversion and that decisions are insensitive (robust) to the precise assignment of prior probabilities. This holds independent from whether the preference for robustness is global or restricted to local perturbations around some reference model.
\end{abstract}

JEL Classification: D81

Keywords: robust decision theory, uncertainty aversion, risk aversion 


\section{Introduction}

In recent years robust or maxmin decision theory has been put forward as an alternative to standard Bayesian decision theory in macroeconomics (e.g. Hansen and Sargent (2000), (2001)). ${ }^{1}$

The key idea behind robust decision theory is that agents might face uncertainty that they cannot quantify in terms of prior probabilities because 'too little is known' to do so.

Without prior probabilities Bayesian decisions are not defined. Robust decision theory fills this gap by postulating that any action is evaluated according to the worst outcome that it can generate among the uncertain states to which prior probabilities cannot be assigned, see Gilboa and Schmeidler (1989) for an axiomatization.

A key motivation for introducing robust decision makers into macroeconomic models is that such models can explain behavior that seems not to be rational from a Bayesian perspective and thereby improve the descriptive performance of otherwise standard macroeconomic models. Hansen et al. (1999), for example, show that a slight preference for robustness can explain a substantial part of the observed equity premiums.

Despite its increasing popularity in applied macroeconomics (e.g. Onatski and Stock (2000), Tetlow and von zur Muehlen (2001)), the relation of robust decision theory to standard Bayesian decision theory seems to have received little attention. At the same time, it seems important to understand the links between the two problems since they might inform us in which ways robust decision makers may alter and improve the descriptive performance of macroeconomic models. Moreover, possible links are potentially informative about how to compute robust decisions in applications.

The present paper shows that robust decision problems can be interpreted in terms of the limit of a sequence of Bayesian decision problems. For a simple class of robust decision problems, I show that there is a sequence of Bayesian

\footnotetext{
${ }^{1}$ I use the term robust decision theory synonymous to the term 'maxmin decision theory', as put forward by Gilboa and Schmeidler (1989). Hansen et. al. (2002) have shown how these two classes of problems can be linked.
} 
decision problems with ever increasing risk aversion that has the property that the associated optimal decisions converge to the optimal robust decision.

Convergence is robust to the precise assignment of prior probabilities by the Bayesian as long as strictly positive probability is assigned to all states over which the robust decision faces unquantifiable uncertainty. This suggests the following Bayesian interpretation of robust decision theory: it represents the choice of a particular objective function that has the property that optimal Bayesian decisions are insensitive (or robust) to many different priors.

These results hold not only when the desire for robustness is of a global nature but also if desired robustness is locally restricted to some small set of perturbations around a reference model.

Besides ever increasing risk-aversion, the sequence of Bayesian decision problems has a second interesting property: utility fails to be time separable even if the objective function of the robust decision maker (seemingly) displays such time separability. This property emerges because the worst case evaluated by the robust decision maker depends on the full decision vector and not only on the decision of a single period.

The next section introduces the decision problem and describes the robust and Bayesian approach to its solution. Section 3 derives the convergence result which is illustrated in section 3.1 with the help of a simple example. Section 4 extends the setup to infinite dimensional decision problems with discounting. An appendix collects the proofs.

\section{Bayesian and Robust Decision Problems}

Consider a decision maker whose objective can be described by a simple loss function that depends on a decision vector $x \in R^{n}$ and an unknown state of the world $s$ :

$$
L(x, s)
$$

$L(\cdot, s)$ is assumed to be twice continuously differentiable and strictly convex for all $s$. The state of the world $s$ is assumed to belong to some finite and known set $\Omega_{s}=\left\{s_{1}, \ldots s_{I}\right\}$ and the decision is assumed to belong to a compact and convex set $\Omega_{x} \subset R^{n}$ of feasible decisions. 
While the state $s$ entering the loss function (1) may be interpreted literally as a state of nature, it is more interesting to interpret $s$ as indexing probability distributions over random events. The latter implies that each $s$ is associated with a different economic model about the underlying stochastic process, which is an interpretation more in line with the recent literature on robust control in macroeconomics.

First, consider a Bayesian decision maker. Based on Savage's axioms such a decision maker can construct subjective prior probabilities $p_{i}(i=1, \ldots n)$ that describe the likelihood with which the decision maker believes that state $s_{i}$ will realize.

Given these priors a Bayesian acts to

$$
\min _{x \in \Omega_{x}} E[L(x, s)]=\min _{x \in \Omega_{x}} \sum_{i=1}^{I} L\left(x, s_{i}\right) p_{i}
$$

Next, consider what has been called a robust decision maker who cannot assign meaningful priors to the realization of the state $s$. The inability to assign prior probabilities might be due to a failure of some of Savage's axioms, e.g. if there is no random variable with uniform distribution that allows for the calibration of probabilities.

Uncertainty that cannot be quantified in terms of subjective probabilities has been called Knightian uncertainty in the literature. The existence of Knightian uncertainty opens many possible ways for modeling the decision problem. One intuitive way, suggested by Blinder (1998), is to simply average over the states of the world. The resulting decision problem would be equivalent to a Bayesian decision problem with $p_{i}=\frac{1}{I}(i=1, \ldots, I)$.

The most widely advocated method to model decisions in the presence of Knightian uncertainty is to let the decision maker choose the action $x$ that minimizes the maximum possible loss associated with $x$. In mathematical terms

$$
\min _{x \in \Omega_{x}} \max _{s \in \Omega_{s}} L(x, s)
$$

Let $x_{r}^{*}$ denote the solution to the minimization part of problem (3). An axiomatic formulation for such a decision theory has been given by Gilboa and Schmeidler (1989). 
When $\Omega_{s}$ contains the states of the world in a primitive sense, then equation (3) implies that the decision maker has a preference for global robustness, as an action $x$ is evaluated with respect to all possible outcomes. For the case where $\Omega_{s}$ indexes a set of probability distributions (economic models), equation (3) allows for a preference for local robustness, as the decision maker seeks only to be robust with respect to models contained in $\Omega_{s}$.

It is useful to rewrite the robust decision problem as follows:

$$
\begin{gathered}
\min _{x \in \Omega_{x}} R(x) \quad \text { with } \\
R(x) \equiv \sum_{i=1}^{I} L\left(x, s_{i}\right) I\left(x, s_{i}\right)
\end{gathered}
$$

where $I\left(x, s_{i}\right)$ is an indicator function that is equal to one if $s_{i}$ is a maximizer of $L\left(x, s_{i}\right)$ and that is equal to zero otherwise. ${ }^{2}$ Rewriting the robust objective in this way helps to highlight the relation to the Bayesian problem (2).

The indicator functions appearing in (4) look almost like 'prior probabilities' of the robust decision maker. These 'robust priors' put all probability weight on the worst state associated with a given decision $x$. Since this worst state may shift with $x$, the 'prior' of the robust decision maker may shift with the chosen decision. This is a major difference to Bayesian priors.

Given the previous observation, there exists an immediate equivalence between robust and Bayesian decisions, as pointed out by Chamberlain (2000) and Hansen et al. (2002): if the Bayesian's priors put all probability weight on the worst state associated with the robust decision, then the optimal Bayesian decision is identical to the robust decision. Note, however, that these priors need not be rational from a Bayesian perspective.

Instead of choosing the Bayesian's priors, this paper seeks to choose an objective function for the Bayesian problem to achieve an equivalence between the optimal Bayesian and robust decisions that holds (almost) independent from the prior probabilities assigned by the Bayesian. This is done in the next section.

\footnotetext{
${ }^{2}$ If there are several maximizers I define the indicator function to be 1 only for the state with the lowest index $i$.
} 


\section{Linking Bayesian and Robust Decision Prob- lems}

The objective of this section is to establish a link between the Bayesian and the robust decision problems described in the previous section. The main idea is to change the objective function of the Bayesian decision problem in a way that the Bayesian's objective will have the same minimum as the robust objective.

Since the Bayesian's loss function depends on the action $x$, altering the loss function is a back-door through which one can cause the Bayesian to behave as if her priors were changing across actions. In particular, if the Bayesian was to maximize a transformed loss function $T(L(x, s))$ with the property that

$$
T(L(x, s))=L(x, s) \cdot \frac{I(x, s)}{p_{s}}
$$

where $p_{s}$ is the prior probability for state $s$, then the Bayesian problem would be identical to the robust decision problem:

$$
\begin{aligned}
& \min _{x \in \Omega_{x}} E[T(L(x, s))] \\
& =\min _{x \in \Omega_{x}} \sum_{i=1}^{I} L\left(x, s_{i}\right) \frac{I\left(x, s_{i}\right)}{p_{i}} p_{i} \\
& =\min _{x \in \Omega_{x}} R(x)
\end{aligned}
$$

Of course, such a transformed 'loss function' is not a loss function in the strict sense since it depends on prior probabilities.

Given that direct equivalence between the two problems requires a Bayesian loss that depends on priors, the strategy is to construct a sequence of transformed loss functions $T^{k}(L(x, s))$ for the Bayesian problem with the property that these transformed loss functions are independent of the prior. At the same time the solution to

$$
\min _{x \in \Omega_{x}} E\left[T^{k}(L(x, s))\right]
$$

which is denoted by $x_{k}^{*}$ should converge to the robust solution $x_{r}^{*}$ as $k$ increases without bound, i.e.

$$
\lim _{k \rightarrow \infty}\left\|x_{k}^{*}-x_{r}^{*}\right\|=0
$$


Define the following sequence of transforming functions $T^{k}(\cdot):^{3}$

$$
T^{k}(L)=e^{k L}
$$

Since $T^{k}(\cdot)$ is increasingly convex as $k$ increases, a Bayesian with objective $T^{k}(L)$ will become increasingly risk averse in terms of the coefficient of absolute risk aversion. As a result, the value of the transformed loss $T^{k}(L)$ increases disproportionately with the size of the loss $L$.

Intuitively, this implies that the largest of all losses $L$ associated with some action $x$ obtains increasing relative weight. This should move the solution to the Bayesian decision problem closer and closer to the robust solution. Proposition 1 below confirms this intuition:

Proposition 1 Let $x_{k}^{*}$ denote the solution to the transformed Bayesian decision problem (6) with prior probabilities $p_{i}>0(i=1, \ldots n)$. Let $x_{r}^{*}$ denote the solution to the robust decision problem (4). Then

$$
\lim _{k \rightarrow \infty}\left\|x_{k}^{*}-x_{r}^{*}\right\|=0
$$

The proof of proposition 1 can be found in the appendix. Proposition 1 shows that robust decisions can be interpreted as decisions of a Bayesian with an infinite degree of risk-aversion and arbitrary strictly positive priors over the domain to which the robust decision maker cannot assign prior probabilities. In Bayesian terms the desire for robustness represents a choice of a particular objective function, which has the property that optimal decisions are robust to the assignment of prior probabilities.

The next subsection illustrates proposition 1 using a univariate example.

\subsection{An Example}

Consider the following simple loss function, which has been considered amongst others by Brainard (1967) and Onatski (2000):

$$
L(x, s)=\left(s x-\pi^{*}\right)^{2}
$$

The variable $\pi^{*}$ denotes an inflation target pursued by the central bank while $s x$ denotes the inflation rate that results when the decision maker chooses policy

\footnotetext{
${ }^{3}$ The particular sequence $T^{k}$ is just chosen for convenience and other sequences might give the same result.
} 
$x$ and the state of the world is given by $s$. If $x$ is the real interest rate, then the factor $s$ represents the sensitivity of the economy's inflation rate to the real interest rate, a number likely to be unknown to the policy maker. Moreover, the policy maker might be unable to assign probabilities to the various values of $s$.

For simplicity, suppose that the desired target inflation rate is $\pi^{*}=2$ and that there are only two potential multipliers $s_{l}<s_{h}$ with $s_{l}=1$ and $s_{h}=3$.

The loss functions associated for each of these multipliers are shown in figure 1. The dotted line in the graph indicates the maximum loss associated with each action. Figure 1 clearly shows that the robust decision that minimizes the maximum loss is given by $x_{r}^{*}=1 .^{4}$

Suppose a Bayesian central bank assigns equal probability to each of the two multipliers $s_{i}(i=l, h)$. The optimal Bayesian decision is then given by $x^{*}=0.8$.

The Bayesian decision maker reacts less aggressively than the robust decision maker. This is the case because the Bayesian trades off the gains and losses across the different realizations of $s$. At the robust decision $(x=1)$ the loss functions in figure 1 have different absolute slope coefficients depending on the value of $s^{i}(i=l, h)$. Therefore, the Bayesian has an incentive to decrease the interest rate below 1 since the gains made for the realization $s_{h}$ will exceed the losses for realizations $s_{l}$, given the prior probabilities assigned to these states.

When the Bayesian's objective function is subjected to increasingly convex transformations through $T^{k}(\cdot)$ risk aversion increases. This implies that the gains for the state $s_{h}$ will be appreciated less relative to the potential losses for state $s_{l}$. Graphically one can interpret this as figure 1 being scaled in the direction of the $y$-axis with each point being scaled by a factor that is increasing with its distance from the $x$-axis. As a result, the slope of $L\left(x, s_{l}\right)$ to the left of $x=1$ increases much faster than the absolute value of the slope of $L\left(x, s_{h}\right)$ to the left of this point. This pushes the Bayesian decision into the direction of the robust decision.

Figure 2 shows how the Bayesian decision approaches the robust decision as $k$ increases, which suggests a convenient way to calculate (approximately) robust decisions. ${ }^{5}$

\footnotetext{
${ }^{4}$ Since there is no uncertainty about the sign of the parameters $s$ the optimal robust decision coincides with the optimal decision under certainty equivalence, as noted by Onatski (2000).

${ }^{5}$ The speed of convergence will depend, amongst other things, on the Bayesian's prior.
} 


\section{Extension}

While the loss function considered so far assumed a finite dimensional decision vector, macroeconomists tend to use infinite horizon models with infinite dimensional decision vectors. In this section we show that the results of the previous section extend in a natural way to the infinite horizon problems with discounting.

Consider the following loss function

$$
L(x, s)=\sum_{t=0}^{\infty} \beta^{t} l\left(x_{t}, s\right)
$$

where $x_{t} \in R^{n}$ denotes the period $t$ decision, the vector $x=\left(x_{0}^{\prime}, x_{1}^{\prime}, \ldots\right)^{\prime}$ the stacked period decisions, and $\beta<1$ a discount factor. The period loss function $l(\cdot, s)$ is assumed to be strictly convex and twice continuously differentiable for all $s$. The period decision $x_{t}$ must be chosen from a compact and convex set of feasible decisions $\Omega_{t}$ that might depend on past decisions. Furthermore, there is a compact set $\Omega_{x} \subset R^{n}$ such that $\Omega_{t} \subset \Omega_{x}$ for all $t$.

The robust decision maker minimizes

$$
\min _{\left\{x_{t} \mid x_{t} \in \Omega_{t}\right\}} \max _{s \in \Omega_{s}} \sum_{t=0}^{\infty} \beta^{t} l\left(x_{t}, s\right)
$$

To construct the transformed Bayesian problem it might seem natural at first to transform the period loss function $l(\cdot, \cdot)$ to preserve the time separability of the objective function, e.g. to let the Bayesian minimize

$$
\min _{\left\{x_{t} \mid x_{t} \in \Omega_{t}\right\}} \sum_{i=1}^{I} \sum_{t=0}^{\infty} \beta^{t} e^{k l\left(x_{t}, s_{i}\right)} p_{i}
$$

However, the solution to this problem will not necessarily converge to the solution of the robust decision problem as $k$ increases without bound. This is the case because a marginal change of some decision might have its strongest impact for a state $s_{i}$ that differs from the worst-case state $s_{r}^{*}$ associated with the robust decision. When, in addition, the sign of the utility change for $s_{i}$ is opposite to the sign of the utility change for $s_{r}^{*}$, then the Bayesian decisions for (8) fails to converge as $k \rightarrow \infty$. This is illustrated in the following example.

Convergence will be slower, the less weight is attached to the worst state associated with the robust decision. 
Example 2 Let the optimal robust decision be given by $x_{r}^{* \prime}=\left(x_{r, 0}^{* \prime}, x_{r, 1}^{* \prime}, \ldots\right)$ and the state $s_{i}(i=1,2)$ that maximizes the loss for this and any neighboring decisions is given by $s_{1}$. Next consider the decision

$$
x^{\prime}=x_{r}^{* *}+\left(d^{\prime}, 0,0,0 \ldots\right)
$$

which is equal to $x_{r}^{*}$, except for the first period. Suppose that altering the decision from $x_{r}^{*}$ to $x$ causes the loss in period zero to increase by $\gamma_{1}>0$ units in state $s_{1}$. This causes $x$ to be suboptimal for the robust decision maker.

Next, consider a Bayesian decision maker with objective (8) who considers a deviation from $x_{r}^{*}$ to $x$. The change $\Delta_{k}$ in the first period loss is given by

$$
\Delta_{k}=\left(e^{k\left(l\left(x_{r, 0}^{*}, s_{1}\right)+\gamma_{1}\right)}-e^{k l\left(x_{r, 0}^{*}, s_{1}\right)}\right) p_{1}+\left(e^{k\left(l\left(x_{r, 0}^{*}, s_{2}\right)+\gamma_{2}\right)}-e^{k l\left(x_{r, 0}^{*}, s_{2}\right)}\right) p_{2}
$$

where $\gamma_{2}=l\left(x_{r, 0}^{*}+d, s_{2}\right)-l\left(x_{r, 0}^{*}, s_{2}\right)$. Suppose $\gamma_{2}<0$ and $l\left(x_{r, 0}^{*}, s_{2}\right)>$ $l\left(x_{r, 0}^{*}, s_{1}\right)+\gamma_{1}>0$, which cannot be excluded, then

$$
\lim _{k \rightarrow \infty} \Delta_{k}=-\infty
$$

which indicates that a Bayesian with objective function (8) will prefer $x$ to $x_{r^{*}}$ for all sufficiently large $k$.

To obtain a convergence result similar to the one in section 3 one has to define the transformed loss function as

$$
T^{k}(L(x, s))=e^{k\left(\sum_{t=0}^{\infty} \beta^{t} l\left(x_{t}, s_{i}\right)\right)}
$$

and let the Bayesian minimize

$$
\min _{\left\{x_{t} \mid x_{t} \in \Omega_{t}\right\}} \sum_{i=1}^{I} e^{k\left(\sum_{t=0}^{\infty} \beta^{t} l\left(x_{t}, s_{i}\right)\right)} p_{i}
$$

where $p_{i}$ are prior probabilities.

Proposition 3 below shows that, as $k$ increases without bound, the Bayesian solution to problem (11) converges to the robust solution in terms of the following vector norm:

$$
\|x\|_{\beta}=\sum_{t=0}^{\infty} \beta^{t} x_{t}^{\prime} x_{t}
$$


Proposition 3 Let $x_{k}^{*}$ denote the solution to the transformed Bayesian decision problem (11) with prior probabilities $p_{i}>0(i=1, \ldots n)$. Let $x_{r}^{*}$ denote the solution to the robust decision problem (7). Then

$$
\lim _{k \rightarrow \infty}\left\|x_{k}^{*}-x_{r}^{*}\right\|_{\beta}=0
$$

The proof of proposition 3 is identical to the one of proposition 1 with the exception that one has to substitute expressions involving the standard vector norm by the 'discounted' norm (12).

As a final remark, I want to stress that the transformed Bayesian utility function fails to be time separable. ${ }^{6}$ Marginal utility for the Bayesian problem is given by

$$
\frac{\partial E\left[T^{k}(L(x, s))\right]}{\partial x_{t}}=k \beta^{t} \sum_{i} \nabla l\left(x_{t}, s_{i}\right) e^{k\left(\sum_{h=0}^{\infty} \beta^{h} l\left(x_{h}, s_{i}\right)\right)} p_{i}
$$

Since this expression does not converge for $k \rightarrow \infty$, consider the ratio of marginal utilities instead:

$$
\frac{\frac{\partial E\left[T^{k}(L(x, s))\right]}{\partial x_{t}}}{\frac{\partial E\left[T^{k}(L(x, s))\right]}{\partial x_{t+j}}}
$$

The limit of (14) for $k \rightarrow \infty$ depends on the states $s$ that maximize $\sum_{h=0}^{\infty} \beta^{h} l\left(x_{h}, s\right)$, where the latter expression is the term showing up in the exponent of (13). Since the states that maximize this expression depend on the whole decision vector $x$, a decision change in some period other than $t$ or $t+j$ may well alter this ratio.

\section{Appendix}

This section proves proposition 1 . Rename states $s$ such that at $x_{r}^{*}$

$$
L\left(x_{r}^{*}, s_{1}\right) \geq L\left(x_{r}^{*}, s_{2}\right) \geq \ldots \geq L\left(x_{r}^{*}, s_{I}\right)
$$

and let

$$
\Omega_{\max }=\left\{i \mid L\left(x_{r}^{*}, s_{i}\right)=L\left(x_{r}^{*}, s_{1}\right)\right\}
$$

I first prove the following auxiliary result:

\footnotetext{
${ }^{6}$ The subsequent arguments assume a non-atomistic decision maker who takes into account that the maximizing states are a function of his/her own decision $x$. See section 6 in Hansen et al. (2002) for further discussion.
} 
Lemma $4 \forall \delta>0$ sufficiently small $\forall d \in R^{n}$ with $\|d\|=\delta \exists \varepsilon>0$ independent of $d$ and a state $i \in \Omega_{\max }$ s.t.

$$
L\left(x_{r}^{*}+d, s_{i}\right)-L\left(x_{r}^{*}, s_{i}\right)>\varepsilon
$$

Proof of lemma 4:. The difference can be expressed as

$$
L\left(x_{r}^{*}+d, s_{i}\right)-L\left(x_{r}^{*}, s_{i}\right)=\nabla L\left(x_{r}^{*}, s_{i}\right) d+d^{\prime} \nabla^{2} L\left(x_{r}^{*}, s_{i}\right) d+O(3)
$$

where $O(3)$ is a third order approximation error. Consider the first order term: From the optimality of $x_{r}^{*}$ follows that

$$
\nabla L\left(x_{r}^{*}, s_{i}\right) d \geq 0
$$

for some $i \in \Omega_{\max }$. Next, fix such an $i$ and consider the second order term. Since $\nabla^{2} L\left(x_{r}^{*}, s_{i}\right)$ is normal and positive definite, we have

$$
\nabla^{2} L\left(x_{r}^{*}, s_{i}\right)=U_{i}^{\prime} D_{i} U_{i}
$$

where $U_{i}$ is unitary and

$$
D_{i}=\operatorname{diag}\left(\lambda_{i, 1} \ldots \lambda_{i, n}\right)
$$

with $\lambda_{i, j}>0$ being the eigenvalues of $\nabla^{2} L\left(x_{r}^{*}, s_{i}\right)$. Then defining $\lambda_{i, \min }=$ $\min _{j} \lambda_{i, j}$

$$
\begin{aligned}
d^{\prime} \nabla^{2} L\left(x_{r}^{*}, s\right) d & =d^{\prime} U_{i}^{\prime} D_{i} U_{i} d \\
& \geq \lambda_{i, \min } d^{\prime} U_{i}^{\prime} U_{i} d \\
& =\lambda_{i, \min } d^{\prime} d \\
& =\lambda_{i, \min } \delta
\end{aligned}
$$

Letting $\lambda_{\min }=\min _{i \in \Omega_{\max }} \lambda_{i, \min }$ it follows from (15), (??), and (19) that

$$
L\left(x_{r}^{*}+d, s_{i}\right)-L\left(x_{r}^{*}, s_{i}\right) \geq \lambda_{\min } \delta^{2}+O(3)
$$

Choosing $\delta$ sufficiently small the third order approximation error can be made arbitrarily small, e.g. smaller than $\frac{\lambda_{\min } \delta}{2}$, then choosing $\varepsilon=\frac{\lambda_{\min } \delta}{2}$ establishes the claim.

Next, normalize the transformed objective of the Bayesian decision maker (6) as follows

$$
\bar{L}^{k}(x)=\frac{1}{e^{k L\left(x_{r}^{*}, s_{1}\right)}} \sum_{i=1}^{I} e^{k L\left(x, s_{i}\right)} p_{i}
$$


Maximizing (20) delivers the same solution as maximizing (6). The limit of $\bar{L}^{k}\left(x_{r}^{*}\right)$ for $k \rightarrow \infty$ exists and is given by:

$$
\lim _{k \rightarrow \infty} \bar{L}^{k}\left(x_{r}^{*}\right)=\sum_{i \in \Omega_{\max }} p_{i}
$$

Next, consider $\bar{L}^{k}\left(x_{r}^{*}+d\right)$ with $d \in R^{n}$ and $\|d\|=\delta, \delta$ sufficiently small. From lemma 4 and (20) it follows that

$$
\bar{L}^{k}\left(x_{r}^{*}+d\right)>\frac{e^{k\left(L\left(x_{r}^{*}, s_{1}\right)+\varepsilon\right)}}{e^{k L\left(x_{r}^{*}, s_{1}\right)}} p_{\min }
$$

where $p_{\min }=\min _{i} p_{i}$. Therefore, there exists a $\bar{k}<\infty$ such that for all $k>\bar{k}$

$$
\bar{L}^{k}\left(x_{r}^{*}+d\right)>\bar{L}^{k}\left(x_{r}^{*}\right)
$$

From the strict convexity of $\bar{L}^{k}(\cdot)$ it follows that the minimum $x_{k}^{*}$ of $\bar{L}^{k}(\cdot)$ must be within distance $\delta$ from $x_{r}^{*}$ for all $k>\bar{k}$, which establishes the claim.

\section{References}

Blinder, Alan, Central Banking in Theory and Practice, MIT Press, 1998.

Chamberlain, Gary, "Econometric Applications of Maxmin Expected Utility," Journal of Applied Econometrics, 2000, 15, 625-644.

Gilboa, Izhak and David Schmeidler, "Maxmin Expected Utility Theory with Non-Unique Prior," Journal of Mathematical Economics, 1989, 18, $141-153$.

Hansen, Lars P. and Thomas J. Sargent, "Wanting Robustness in Macroeconomics," 2000. Standord University, mimeo.

Hansen, L.P., T.J. Sargent, and T. Tallarini, "Robust Permanent Income and Pricing," Review of Economic Studies, 1999, 66, 873-907.

_ _ _ G.A. Tirmuhambetova, and N. Williams, "Robustness and Uncertainty Aversion," 2002. Stanford University Mimeo.

Onatski, Alexei, "Minimax Analysis of Monetary Policy under Model Uncertainty," 2000. Harvard University, Dept. of Economics, mimeo.

and James Stock, "Robust Monetary Policy under Model Uncertainty in a Small Model of the U.S. Economy," NBER Working Paper 7490, 2000. 
Sargent, T.J. and L.P. Hansen, Robust Control and Filtering for Macroeconomics, Stanford University book mimeo, 2001.

Tetlow, Robert and Peter Von Zur Muehlen, "Robust Monetary Policy with Misspecified Models: Does Model Uncertainty Always Call for Attenuated Policy?," 2001. Federal Reserve Board mimeo. 


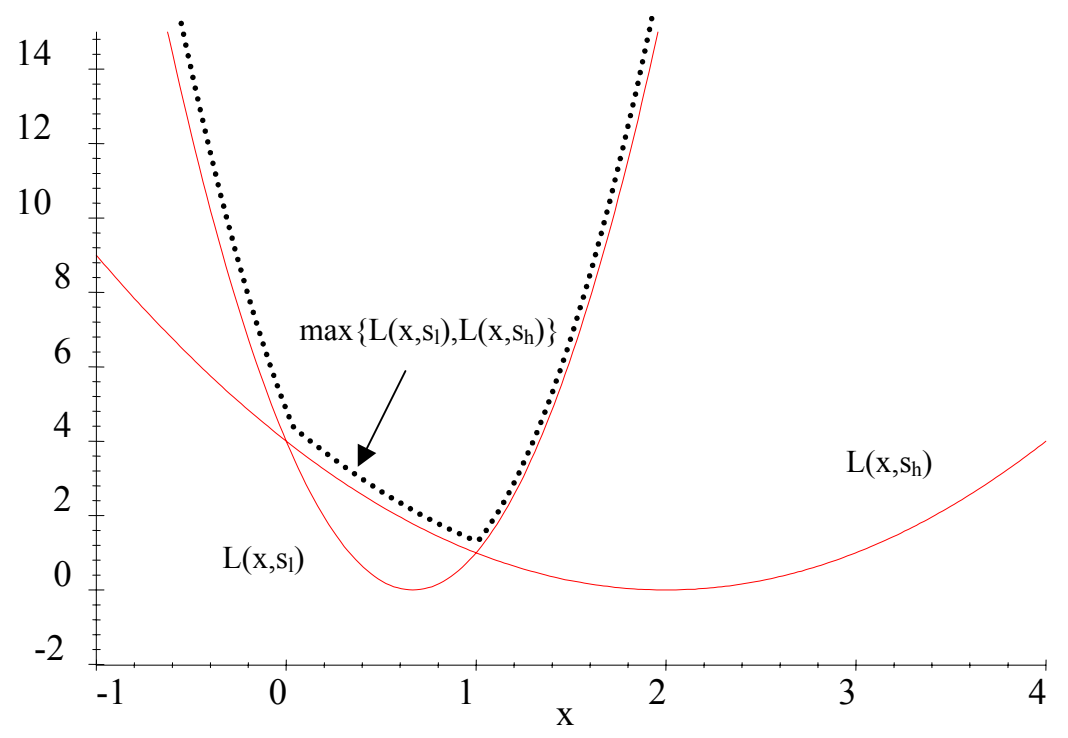

Figure 1: Loss functions

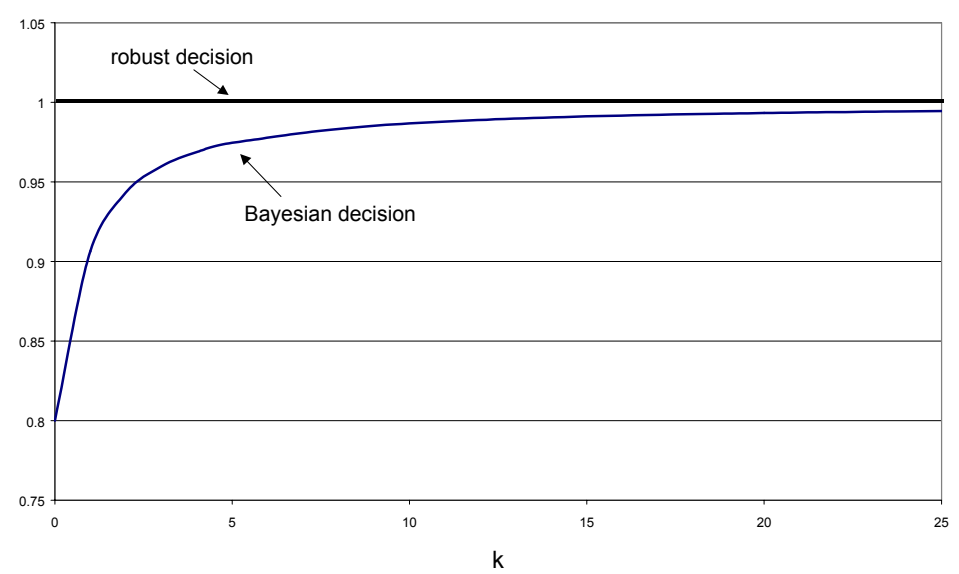

Figure 2: Bayesian decision as a function of the parameter $k$ 


\section{CFS Working Paper Series:}

\begin{tabular}{|c|c|c|}
\hline No. & Author(s) & Title \\
\hline 2002/07 & $\begin{array}{l}\text { Werner Neus } \\
\text { Uwe Walz }\end{array}$ & $\begin{array}{l}\text { Exit Timing of Venture Capitalists in the Course } \\
\text { of an Initial Public Offering }\end{array}$ \\
\hline 2002/08 & $\begin{array}{l}\text { Andreas Bascha } \\
\text { Uwe Walz }\end{array}$ & $\begin{array}{l}\text { Financing Practices in the German Venture } \\
\text { Capital Industry } \\
\text { An Empirical Assessment }\end{array}$ \\
\hline 2002/09 & Marco Hoeberichts & The Credibility of Central Bank Announcements \\
\hline $2002 / 10$ & $\begin{array}{l}\text { Markus Haas } \\
\text { Stefan Mittnik } \\
\text { Marc S. Paolella }\end{array}$ & Mixed Normal Conditional Heteroskedasticity \\
\hline $2002 / 11$ & Elke Hahn & $\begin{array}{l}\text { Core Inflation in the Euro Area: } \\
\text { An Application of the } \\
\text { Generalized Dynamic Factor Model }\end{array}$ \\
\hline $2002 / 12$ & $\begin{array}{l}\text { Yunus Aksoy } \\
\text { Hanno Lustig }\end{array}$ & $\begin{array}{l}\text { On the Short and Long Term Real Effects of } \\
\text { Nominal Exchange Rates }\end{array}$ \\
\hline $2002 / 13$ & Andreas A. Jobst & $\begin{array}{l}\text { Collateralised Loan Obligations (CLOs) - } \\
\text { A Primer }\end{array}$ \\
\hline $2002 / 14$ & Andreas A. Jobst & $\begin{array}{l}\text { The Pricing Puzzle: The Default Term Structure } \\
\text { of Collateralised Loan Obligations }\end{array}$ \\
\hline $2002 / 15$ & Athanasios Orphanides & $\begin{array}{l}\text { Activist Stabilization Policy and Inflation: } \\
\text { The Taylor Rule in the 1970s }\end{array}$ \\
\hline 2003/01 & Klaus Adam & $\begin{array}{l}\text { Learning to Forecast and Cyclical Behavior of } \\
\text { Output and Inflation }\end{array}$ \\
\hline 2003/02 & Klaus Adam & $\begin{array}{l}\text { On the Relation between Robust and Bayesian } \\
\text { Decision Making }\end{array}$ \\
\hline
\end{tabular}

Copies of working papers are available at the Center for Financial Studies or can be downloaded (http://www.ifk-cfs.de). 